\title{
Experimental Study of a Linear Fresnel Collector with Triangular-Cavity Absorber
}

\author{
Jinghui Song ${ }^{1, \mathrm{a}}$, Jishuai $\mathrm{Ma}^{2, \mathrm{~b}^{*}}$, Fangyong $\mathrm{Li}^{1}, \mathrm{Yu} \mathrm{Chen}^{2}$, Yanjun $\mathrm{Dai}^{2}$ \\ ${ }^{1}$ Electric Power Research Institute of Guangdong Power Grid Co., Ltd., Guangzhou 510080, China; \\ ${ }^{2}$ Institute of Refrigeration and Cryogenics, Shanghai Jiao Tong University, Shanghai 200240, China \\ aemail: songjinghui2006@163.com, bemail: biansaixingyin@163.com
}

Key words: Fresnel collector; cavity absorber; performance test; thermal efficiency

Abstract: A Fresnel solar collector with triangular cavity absorber is introduced and the performance tests are carried on. The Fresnel collector is consisted of a cavity absorber with tube rows, a mirror field with flat glass, and the trace system in homotaxial manner. According to relative technical standards, the transient method is used in the performance tests. Based on the test results, the no-load coefficient, the heat loss coefficient and the thermal efficiency of the collector are calculated and analyzed. The result shows that the thermal efficiency is up to $36.6 \%$ and the heat loss coefficient is $110 \mathrm{~W} / \mathrm{m}$.

\section{Introduction}

The Fresnel linear focus technology have been proposed in the 1960s, which can produce 100 $250{ }^{\circ} \mathrm{C}$ stable heat source, providing heat of middle temperature for industry processes. Compared with the trough linear focus technology, DRMills ${ }^{[1]}$ noted that Fresnel collector costs only about $50 \%-60 \%$ of the latter per square meter. But the disadvantage is that the heat efficiency is low, such as trough collector's efficiency can reach 70-80\%, while the Fresnel collector only about 50\% $-60 \%$. Therefore, with respect to the trough collectors, Fresnel collector needs a larger area.

In Fig. 1, the Fresnel collector consists of mirrors of plurality of rows, each row of which tracks the sun in a certain angle and reflects sunlight into the absorber. The absorber transfers heat to the the working medium and heats it. Since the reflection area is several tens of times larger than the absorption area, the collector make the solar energy of low density into solar energy of high density.

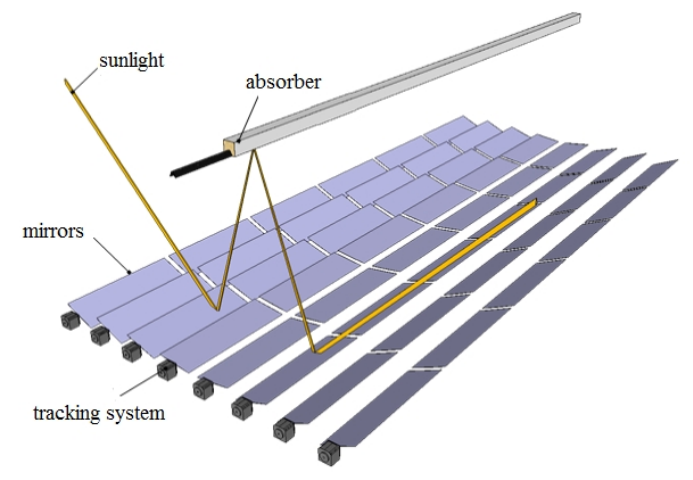

Fig.1 The 3D model of Linear Fresnel solar collector

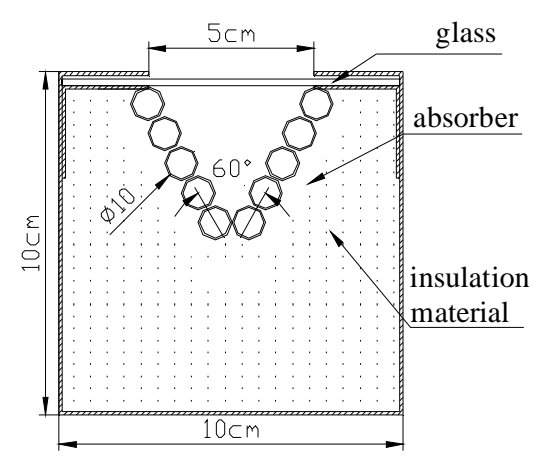

Fig.2 Triangle cavity absorber

This paper describes the construction and property test of the Fresnel collector with a triangular cavity absorber (Fig.2). First of all, according to the design theory, the collector's designs, including the mirrors' arrangement, size and tracking manner are confirmed. Then the no load performance test and transient efficiency test are carried on. In comparison with the trough collector, the Fresnel collector's efficiency is about 10 percent lower. However, because of the easy production, low cost, 
easy operation and maintenance, the Fresnel collector is still applied in the middle temperature field of solar energy utilization.

\section{Construction of the linear Fresnel collector}

The Fresnel collector aims to provide heat of $150 \sim 180{ }^{\circ} \mathrm{C}$, and according to Zhang Liying's ${ }^{\text {[2] }}$ calculations, its concentration ratio is between 40 to 50 which will meet the requirements. We determine it to be 48 .

\section{Absorber}

As for the selection of the absorber, we choose the triangular cavity absorber instead of the vacuum tube used in the trough collector referring to the study of Zhai Hui ${ }^{[3]}$. Taking into account that the working medium is not limited to HTF oil, possibly can be water, vapor and two-phase flow, we choose the absorber in the form of tube rows one is able to bear higher pressure (shown in Fig2). Considering the structure of the triangular cavity absorber, secondary concentration form is not used. The absorbed is mainly consisted of the brass tubes, the stainless steel cover, the glass cover and the insulation layer. Physical parameters of the components are shown in Table 1.

Table 1 Physical parameters of the cavity

\begin{tabular}{lcccc}
\hline Materials & $\rho / \mathrm{kg} \cdot \mathrm{m}^{-3}$ & $\lambda / \mathrm{W} \cdot \mathrm{m}^{-1} \cdot \mathrm{K}^{-1}$ & $\mathrm{C}_{\mathrm{p}} / \mathrm{J} \cdot \mathrm{kg}^{-1} \cdot \mathrm{K}^{-1}$ & $\varepsilon$ \\
\hline Copper (selective coating) & 8978 & 387.6 & 381 & 0.5 \\
Aluminum & 2710 & 236 & 902 & 0.1 \\
Stainless steel & 7830 & 14.7 & 460 & 0.1 \\
Glass & 2719 & 0.65 & 871 & 0.85 \\
Insulation material & 41 & 0.035 & 35 & - \\
\hline
\end{tabular}

\section{Mirror field}

Because the absorber doesn't have a secondary condenser, and the light entrance width is $5 \mathrm{~cm}$, the width of the mirror should be generally $30 \mathrm{~cm}$ or more. And the mirror is curved with a certain radian so that the light can be reflected to the light entrance of the absorber. To guarantee the concentration ratio, the total width of the mirrors is set to $240 \mathrm{~cm}$.

\section{Tracking Systems}

As for the tracking form of the mirrors, considering that the multi-row mirrors will track the sun at the same time, we choose the form of a single row of independent driven system, of which each mirror has a small step motor tracking.

In summary, the final basic design parameters of the Fresnel collectors are shown in Table 2.

Table 2 Basic design parameter of the Fresnel collector

\begin{tabular}{ll|ll}
\hline Events & Parameters & Events & Parameters \\
\hline Work temperature & $100 \sim 250^{\circ} \mathrm{C}$ & Type of the mirror & Slightly curved pattern \\
& & Total width of mirrors & $240 \mathrm{~cm}$ \\
Concentration ratio & 48 & Numbers of mirrors & 8 \\
Type of absorber & Triangle-cavity absorber & tracking form & Homotaxial tracking \\
Size of absorber & Referring to Fig. 2 & trang &
\end{tabular}




\section{Test system of the Fresnel collector}

The measuring the collector's efficiency curve refers to the standards GB / T4271-2000 and ASHRAE 93-86, in the way of instantaneous measurement. In order to achieve the quasi-steady state conditions, in a time interval condition, the fluctuation of direct solar radiation intensity doesn't exceed $\pm 50 \mathrm{~W} / \mathrm{m}^{2}$, the fluctuation of the inlet temperature of the collector's working fluid is less than $1^{\circ} \mathrm{C}$, the fluctuation of working fluid flow rate is no more than $\pm 1 \%$, the ambient temperature change is less than $1{ }^{\circ} \mathrm{C}$, the ambient temperature variation range is smaller than $30{ }^{\circ} \mathrm{C}$, and the wind speed is not greater than $4 \mathrm{~m} / \mathrm{s}$ throughout the entire testing process.

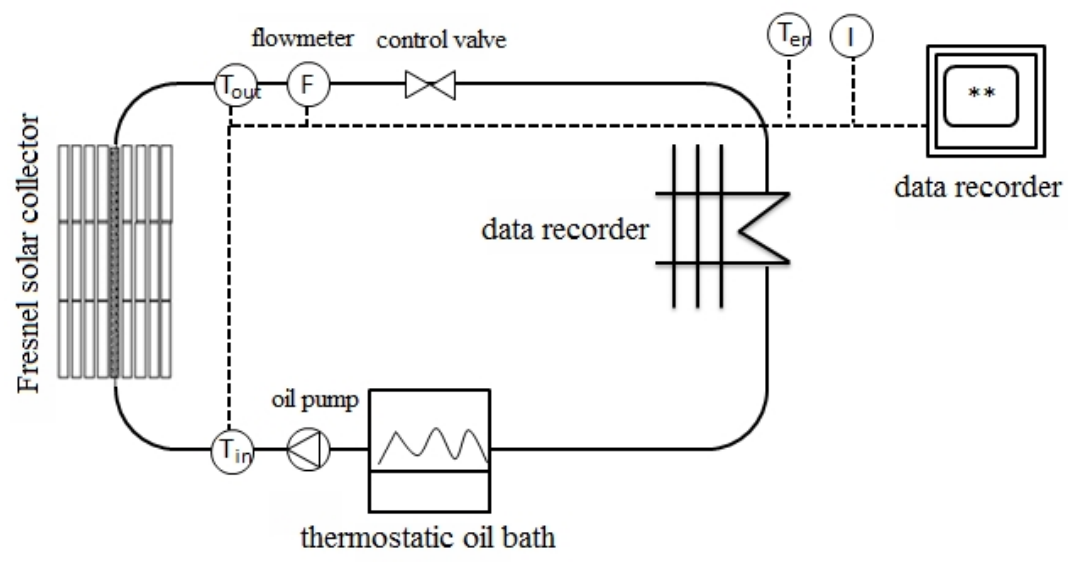

Fig.3 Schematic of the collector's test system

The components of the performance test system are shown in Figure 3. The figure marks the location of each sensor's measuring point. In the experiment system, the temperature is measured by four-wire PT100 platinum resistance measurement, the direct radiation intensity and total radiation intensity is measured by Kipp \& Zonen company's Sun tracker which can automatically realize the sun's two-dimensional tracking, with two full-radiometer and a direct radiometer.

\section{Fresnel line focusing performance test}

\section{No-load test}

To verify the maximum working temperature of the collector, the test carried out no-load performance test. The import and export of the absorber are closed to avoid the impact brought to the test results, caused by the convection between the air within and out the absorber. During the test, the temperature and the solar irradiance at the measuring points are recorded every $1 \mathrm{~min}$. The test time is from 9:40 17:00 in a sunny and breezy day, and the test results are shown in Figure 4. Select the data: total irradiation intensity, direct radiation intensity, ambient temperature, temperature of the cover glass and the absorber's surface, for analysis. The results show that: (1) the average direct radiation intensity is $664.0 \mathrm{w} / \mathrm{m}^{2}$, and the average ambient temperature is $18.6{ }^{\circ} \mathrm{C}$; (2) the temperature of the absorber's surface rises to $260.3{ }^{\circ} \mathrm{C}$ with the collecting time increasing; meanwhile, the temperature of the glass cover also rises up to $60^{\circ} \mathrm{C}$. The changes amplitude of both decrease sequentially in the later period.

\section{Transient test}

During the test, at least 8 measuring points are choosed and the test results are shown in Figure 5. The transient thermal efficiency of the collector is calculated by the following formula, 


$$
\eta_{t}=\frac{n x_{p, w}\left(T_{o u t}-T_{i n}\right)}{A I_{b}}
$$

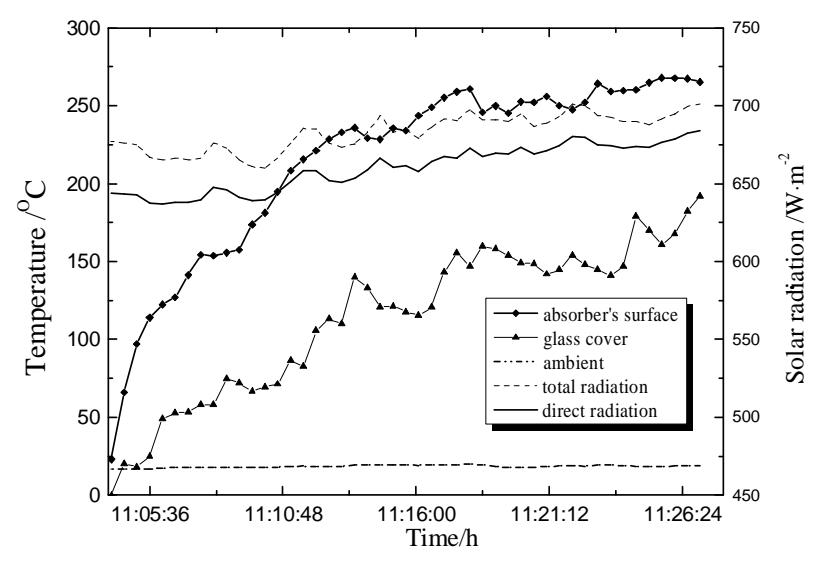

Fig.4 The no-load test of the collector

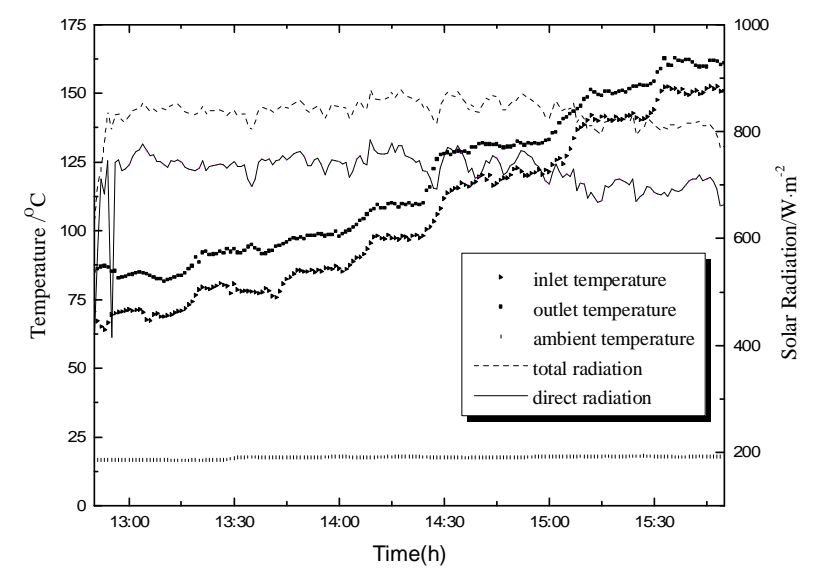

Fig.5 The transient test of the collector

Fig.5, the efficiency curve is fitted by the measured data $T_{s}$ and $\eta_{c}$ using the least square method. During the test, it's sunny, gently breezy, and temperature fluctuates in the vicinity of $16-18^{\circ} \mathrm{C}$. The mass flow rate of HTF is $168 \mathrm{~g} / \mathrm{s}$.

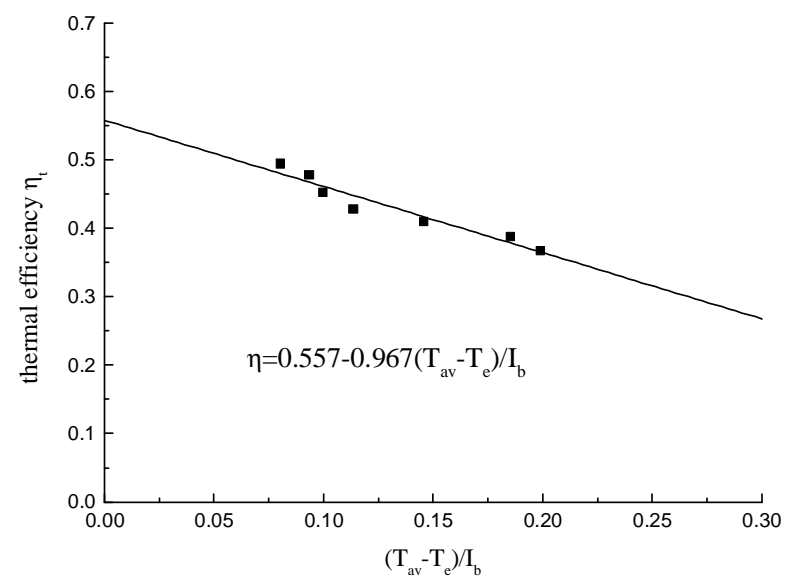

Fig.6 Results of the performance test

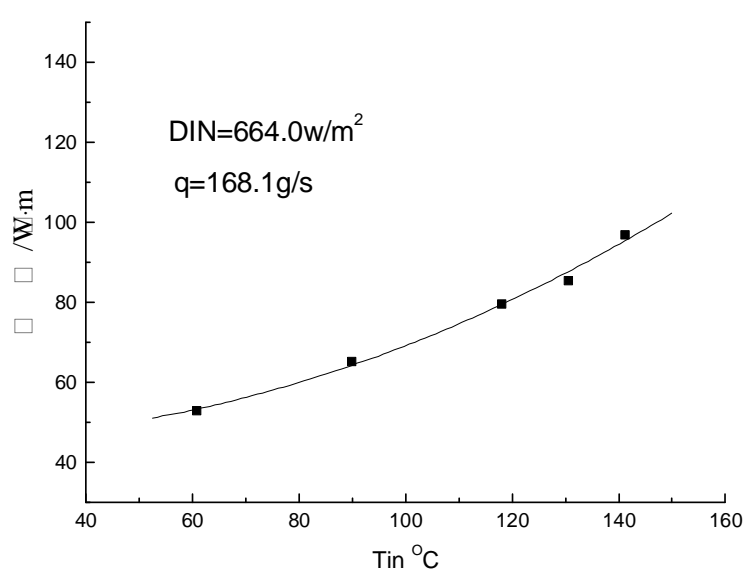

Fig.7 Heat loss coefficient of the absorber

In figure 6 , the intercept of the efficiency curve is the optical efficiency of the system. The average value of the test is $55.7 \%, 11.4 \%$ less than the ideal optical efficiency $67.1 \%$. When the direct radiation intensity is $675.9 \mathrm{~W} / \mathrm{m}^{2}$, the system efficiency can reach $45.2 \%$ at the working temperature $90^{\circ} \mathrm{C}$, and $36.6 \%$ at $150^{\circ} \mathrm{C}$, both slightly lower than expected results.

Figure 7 shows the triangular cavity absorber's heat loss coefficient $\mathrm{U}_{\mathrm{L}, \mathrm{a}}$. According to the experiment results, it is $110 \mathrm{~W} / \mathrm{m}$ at $150^{\circ} \mathrm{C}$, larger than the vacuum absorber.

\section{Summary}

This article mainly introduces the construction and the performance test of the linear Fresnel collector with a triangular cavity absorber. The test results show that the temperature of the HTF rises up to $260.3^{\circ} \mathrm{C}$ within half an hour, and its no-load performance parameters is $0.364^{\circ} \mathrm{C} \cdot \mathrm{m}^{2} / \mathrm{W}$. 
Furthermore, the average value of the test is $55.7 \%, 11.4 \%$ less than the ideal optical efficiency $67.1 \%$. Under the experiment conditions, the system efficiency is $45.2 \%$ at the working temperature $90^{\circ} \mathrm{C}$, and $36.6 \%$ at $150^{\circ} \mathrm{C}$ and the heat loss coefficient is $110 \mathrm{w} / \mathrm{m}$.

\section{References}

[1] D.R.Mills. Project Proposal for a Compact Linear Fresnel Reflector Solar thermal Plant in the Hunter Valley[EB].

[2] Zhang Liying. Thermal analysis of cavity absorber for solar energy heat collector[J]. Journal of engineering thermophysics, 2008, 29(9).

[3] Zhai Hui. Theoretical and experimental investigation of linear concentrating solar collector with cavity absorber[D]. Shanghai: Shanghai Jiao Tong University, 2009.

[4] Bai Tao. Analysis on heat removal factor for cavity absorber of a linear solar concentrator and its utilization[D]. Shanghai: Shanghai Jiao Tong University, 2010.

[5] John A. Duffie, William A. Beckman. Solar Engineering of Thermal Processes[M]. New Jersey: John Wiley \& Sons, Inc., Hoboken. 2013. 